\title{
THE INITIAL LAYER PROBLEM AND INFINITE PRANDTL NUMBER LIMIT OF RAYLEIGH-BÉNARD CONVECTION*
}

\author{
JIANGUO $\mathrm{SHI}^{\dagger}, \mathrm{KE} \mathrm{WANG}^{\ddagger}, \mathrm{AND}$ SHU WANG ${ }^{\S}$
}

\begin{abstract}
In this paper the Initial layer problem and infinite Prandtl number limit of RayleighBénard convection are studied. For the case of ill-prepared initial data infinite Prandtl number limit of the Boussinesq approximation for Rayleigh-Bénard convection is proven by using the asymptotic expansion methods of singular perturbation theory and the classical energy methods. An exact approximating solution with the zero order term and the 1st order term expansion is given and the convergence rates $O\left(\epsilon^{\frac{3}{2}}\right)$ and $O\left(\epsilon^{2}\right)$ are respectively obtained. This improves the result of X. M. Wang [Commun. Pure Appli. Math., LVII(2004), 1265-1282].
\end{abstract}

Key words. Boussinesq approximation, Rayleigh-Bénard convection, initial layer, infinite Prandtl number limit, asymptotic expansions, singular perturbation, classical energy methods

Subject classifications. 35B25, 35B40, 35K57

\section{Introduction}

Rayleigh-Bénard convection of fluid involving heat transfer, and confined by two parallel planes a distance $h$ apart and heated at the bottom plane at the temperature $T_{2}$ and cooled at the top plane at the temperature $T_{1}\left(T_{2}>T_{1}\right)$, can be approximated by the following so-called Boussinesq system in a rotating frame

$$
\begin{aligned}
& \partial_{t} u+(u \cdot \nabla) u+\nabla p+2 \Omega e_{3} \times u=\nu \Delta u+g \alpha e_{3} T, \\
& \nabla \cdot u=0 \\
& \partial_{t} T+(u \cdot \nabla) T=\kappa \Delta T, \\
& \left.u\right|_{z=0, h}=0, \\
& \left.T\right|_{z=0}=T_{2},\left.T\right|_{z=h}=T_{1},
\end{aligned}
$$

where $u$ is the velocity field of the fluid, $p$ is the pressure, $\Omega$ is the rotation rate, $e_{3}$ is the unit upward vector, $\nu$ is the kinematic viscosity, $g$ is the gravity acceleration constant, $\alpha$ is the thermal expansion coefficient, $T$ is the temperature field of the fluid, $\kappa$ is the thermal diffusion coefficient. Here we also impose the periodic boundary conditions in the horizontal directions for simplicity.

This set of equations is much more complex because this dynamic system consists of heat advection-diffusion of the temperature coupled with the incompressible NavierStokes equations via a buoyancy force proportional to the temperature see $[1,3,17$, 19, 20]. Thanks to the physics, one can use the much simplified system to study the problem and hence the simplification of Boussinesq system is highly desirable. To

*Received: August 15, 2006; accepted (in revised version): November 2, 2006. Communicated by Shi Jin.

S. Wang's research was partially supported by the NSFC (Grant no.10471009) and BSFC (Grant no. 1052001) of China, the Educational Ministry of China (Grant no.NCET-04-0203), and the Personal Ministry of China.

${ }^{\dagger}$ Department of Mathematics, Huanghuai College, Zhumadian 463000, Henan Province, P. R. China.

${ }^{\ddagger}$ College of Applied Sciences, Beijing University of Technology Ping Le Yuan100, Chao Yang District, Beijing100022, P. R. China (coco@emails.bjut.edu.cn).

$\S$ College of Applied Sciences, Beijing University of Technology Ping Le Yuan100, Chao Yang District, Beijing100022, P. R. China (wangshu@bjut.edu.cn). 
this end, we will use the nondimensional version. Introducing the suitable scalings, the nondimensinal form of Boussinesq system can be taken as

$$
\begin{aligned}
& \epsilon\left[\partial_{t} u^{\epsilon}+\left(u^{\epsilon} \cdot \nabla\right) u^{\epsilon}\right]+\nabla p^{\epsilon}+\frac{1}{E k} e_{3} \times u^{\epsilon}=\Delta u^{\epsilon}+\operatorname{Rae}_{3} T^{\epsilon}, \\
& \nabla \cdot u^{\epsilon}=0, \\
& \partial_{t} T^{\epsilon}+\left(u^{\epsilon} \cdot \nabla\right) T^{\epsilon}=\Delta T^{\epsilon}, \\
& \left.u^{\epsilon}\right|_{z=0,1}=0 \\
& \left.T^{\epsilon}\right|_{z=0}=1,\left.T^{\epsilon}\right|_{z=1}=0, \\
& u^{\epsilon}(t=0)=u_{0}^{\epsilon}(x, y, z), T^{\epsilon}(t=0)=T_{0}^{\epsilon}(x, y, z)
\end{aligned}
$$

for $(x, y, z) \in G=\mathcal{T}^{2} \times(0,1), t>0$, where $\mathcal{T}=\left(\frac{R^{1}}{2 \pi}\right)^{2}$ is the torus in $R^{2}, \epsilon=\frac{1}{\operatorname{Pr}}, \operatorname{Pr}=\frac{\nu}{\kappa}$ is the Prandtl number, $E k=\frac{\nu}{2 \Omega h^{2}}$ is the Ekman number and $R a=\frac{g \alpha\left(T_{2}-T_{1}\right) h^{3}}{\nu \kappa}$ is the Rayleigh number.

In this paper we are interested in the infinite Prandtl number limit $\operatorname{Pr} \rightarrow \infty$, i.e., the limit $\epsilon \rightarrow 0$, of Rayleigh-Bénard convection (1.1)-(1.6). Formally, setting $\epsilon=0$ in the system (1.1)-(1.5), we arrive at the following infinite Prandtl number system

$$
\begin{aligned}
& \nabla p^{0}+\frac{1}{E k} e_{3} \times u^{0}=\Delta u^{0}+\operatorname{Rae}_{3} T^{0}, \\
& \nabla \cdot u^{0}=0 \\
& \partial_{t} T^{0}+\left(u^{0} \cdot \nabla\right) T^{0}=\Delta T^{0} \\
& \left.u^{0}\right|_{z=0,1}=0 \\
& \left.T^{0}\right|_{z=0}=1,\left.T^{0}\right|_{z=1}=0
\end{aligned}
$$

for $(x, y, z) \in G, t>0$, which can be completed by the initial data

$$
T^{0}(t=0)=T_{0}^{0}(x, y, z),(x, y, z) \in G,
$$

where $T_{0}^{0}(x, y, z)$ is the limit of $T_{0}^{\epsilon}(x, y, z)$ as $\epsilon \rightarrow 0$. But, due to the singularity of perturbation, generally speaking, the limit of $u_{0}^{\epsilon}(x, y, z)$ as $\epsilon \rightarrow 0$ can not be satisfied by the velocity $u^{0}(t=0)$ in the limit system. In fact, restricting the equations (1.7), (1.8) and (1.10) to $t=0$, one gets

$$
\begin{aligned}
& \nabla p^{0}(t=0)+\frac{1}{E k} e_{3} \times u^{0}(t=0)=\Delta u^{0}(t=0)+\operatorname{Rae}_{3} T^{0}(t=0), \\
& \nabla \cdot u^{0}(t=0)=0, \\
& \left.u^{0}\right|_{z=0,1}=0 .
\end{aligned}
$$

This is a stationary Stokes equation with rotation, which implies that the value $u^{0}(t=$ $0)$ is determined by the initial data $T_{0}^{0}$ of the temperature $T^{0}(x, y, z, t)$ by solving the system (1.13)-(1.15). But, the limit $\lim _{\epsilon \rightarrow 0} u_{0}^{\epsilon}\left(\neq u^{0}(t=0)\right)$ can be given arbitrarily and independently of $T_{0}^{0}$. Thus, an initial layer occurs. Hence the infinite Prandtl number limit is a singular limit problem involving an initial layer.

Recently X. M. Wang [19] considered this limit and obtained an effective approximating system; then, by using this effective system and hence avoiding the exact structure of the approximating solution, proved the convergence of Rayleigh-Bénard convection to the infinite Prandtl number limit system in the sense of $L^{2}$-norm with a convergence rate $O(\epsilon)$. The main purpose of this paper is to improve the result 
of X. M. Wang [19] by the asymptotic expansion methods of the singular perturbation theory $[9,10,18,21]$. More precisely, we first obtain the exact structure of the approximating solution with an expansion of the zero order term and the 1st order term, which is very interesting in the application of physics. Then the convergence of this approximating solution is proven and the convergence rate $O\left(\epsilon^{\frac{3}{2}}\right)$ and the optimal convergence rate $O\left(\epsilon^{2}\right)$ are respectively obtained. Our analysis also shows that there appears the mixed two-fast-scaled time and space boundary layer, caused by the initial layer, for the asymptotic expansion with the 2nd order corrector.

It should be pointed out that there have been many results on the global existences and regularities of the suitable weak solution, see [19, 20], on the global existence of smooth solutions, the upper bound problem for infinite Prandtl number system and the related models, see $[1,2,4,6,11,8,14,15,17]$, and on the interesting Nusselt number problem in turbulent flow, see [5] and the references therein. A related rotating Boussinesq model problem in geophysical fluid dynamics has widely been discussed, see [12].

This paper is organized as follows: In section 2 , the precise convergence results are stated. In section 3, the approximating solutions are constructed and the properties of approximating solutions are given. Sections 4 is devoted to the proofs of main convergence results.

\section{Main results}

In this section we state the main results of this paper.

Assume that the initial data have an expansion up to the 1st order as follows

$$
\left(u^{\epsilon}, T^{\epsilon}\right)(t=0)=\left(u_{0}^{0}+\epsilon u_{0}^{1}+u_{0 R}^{\epsilon}, T_{0}^{0}+\epsilon T_{0}^{1}+T_{0 R}^{\epsilon}\right)(x, y, z),
$$

where $u_{0}^{0}, u_{0}^{1}, T_{0}^{0}$ and $T_{0}^{1}$ are all $C^{\infty}(G)$ functions, and $u_{0 R}^{\epsilon}(x, y, z), T_{0 R}^{\epsilon}(x, y, z) \in C^{\infty}(G)$ satisfy $\left\|\left(u_{0 R}^{\epsilon}, T_{0 R}^{\epsilon}\right)(x, y, z)\right\|_{L^{2}(G)} \leq C \epsilon^{2}$ for some positive constant $C$ independent of $\epsilon$.

Take the ansatz as the approximating solution

$$
\begin{aligned}
& \left(u_{a p p}^{\epsilon}, p_{\text {app }}^{\epsilon}, T_{\text {app }}^{\epsilon}\right)(x, y, z, t) \\
= & \sum_{i=0}^{1} \epsilon^{i}\left(u^{i}(x, y, z, t)+\bar{u}^{i}(x, y, z, \tau),\right. \\
& \left.p^{i}(x, y, z, t)+\bar{p}^{i}(x, y, z, \tau), T^{i}(x, y, z, t)+\bar{T}^{i}(x, y, z, \tau)\right),
\end{aligned}
$$

where $\tau=\frac{t}{\epsilon}$ is the fast time variable, the outer functions $\left(u^{i}, p^{i}, T^{i}\right)(x, y, z, t), i=0,1$, is independent of $\epsilon$ while $\left(\bar{u}^{i}, \bar{p}^{i}, \bar{T}^{i}\right)(x, y, z, \tau), i=0,1$, are the initial layer functions near $t=0$. We will discuss in detail the construction of the outer and initial layer functions in the next section, however, we summarize the results here.

First, the outer function $\left(u^{0}, p^{0}, T^{0}\right)(x, y, z, t)$ is determined as the solution of the infinite Prandtl number system (1.7)-(1.12), and $\left(u^{1}, p^{1}, T^{1}\right)(x, y, z, t)$ is the solution of the following linear Pandtl type problem

$$
\begin{aligned}
& \nabla p^{1}+\frac{1}{E k} e_{3} \times u^{1}=\Delta u^{1}+\operatorname{Rae}_{3} T^{1}-\partial_{t} u^{0}-\left(u^{0} \cdot \nabla\right) u^{0}, \\
& \nabla \cdot u^{1}=0 \\
& \partial_{t} T^{1}+\left(u^{0} \cdot \nabla\right) T^{1}+\left(u^{1} \cdot \nabla\right) T^{0}=\Delta T^{1}, \\
& \left.u^{1}\right|_{z=0,1}=0 \\
& \left.T^{1}\right|_{z=0,1}=0 \\
& T^{1}(t=0)=T_{0}^{1}(x, y, z)-\bar{T}^{1}(t=0),
\end{aligned}
$$


where $\bar{T}^{1}(t=0)$ will be determined later(see below (3.22)).

Then, the initial layer functions $\left(\bar{u}^{i}, \bar{p}^{i}, \bar{T}^{i}\right)(x, y, z, \tau), i=0,1$, are determined as the solution of the following problems respectively

$$
\begin{aligned}
& \bar{T}^{0}(x, y, z, \tau)=0, \\
& \partial_{\tau} \bar{u}^{0}+\frac{1}{E k} e_{3} \times \bar{u}^{0}+\nabla \bar{p}^{0}=\Delta \bar{u}^{0}, \\
& \nabla \cdot \bar{u}^{0}=0, \\
& \partial_{\tau} \bar{T}^{1}+\bar{u}^{0} \nabla T_{0}^{0}=0, \\
& \left.\bar{u}^{0}\right|_{z=0,1}=0, \\
& \bar{u}^{0}(\tau=0)=u_{0}^{0}-u^{0}(t=0),\left(\bar{u}^{0}, \bar{T}^{1}\right)(\tau \rightarrow \infty)=0
\end{aligned}
$$

and

$$
\begin{aligned}
& \partial_{\tau} \bar{u}^{1}+\frac{1}{E k} e_{3} \times \bar{u}^{1}+\nabla \bar{p}^{1} \\
& =\Delta \bar{u}^{1}+\operatorname{Rae}_{3} \bar{T}^{1}-\left(\bar{u}^{0} \cdot \nabla\right) u_{0}^{0}-\left(u_{0}^{0} \cdot \nabla\right) \bar{u}^{0}-\left(\bar{u}^{0} \cdot \nabla\right) \bar{u}^{0}, \\
& \nabla \cdot \bar{u}^{1}=0 \\
& \left.\bar{u}^{1}\right|_{z=0,1}=0 \text {, } \\
& \bar{u}^{1}(\tau=0)=u_{0}^{1}-u^{1}(t=0), \bar{u}^{1}(\tau \rightarrow \infty)=0 .
\end{aligned}
$$

The existence of the smooth solution to the above outer function and initial layer function problems will be discussed in the section $\S 3$.

Now we state the main results as follows.

First, we have the convergence rate $O\left(\epsilon^{\frac{3}{2}}\right)$.

Theorem 2.1. Assume that (2.1) holds. Also, assume that $u_{0}^{0}, u_{0}^{1}, T_{0}^{0}, T_{0}^{1} \in C^{\infty}(G)$ satisfy the suitable compatibility conditions like $\left.u_{0}^{0}\right|_{z=0,1}=0, \nabla \cdot u_{0}^{0}=0,\left.T_{0}^{0}\right|_{z=0}=$ $1,\left.T_{0}^{0}\right|_{z=1}=0,\left.u_{0}^{1}\right|_{z=0,1}=0, \nabla \cdot u_{0}^{1}=0,\left.T_{0}^{1}\right|_{z=0,1}=0$, etc. Then, as $\epsilon \rightarrow 0$, for any $0<S<$ $\infty$, we have the following convergence:

$$
\left\|\left(u^{\epsilon}-u_{a p p}^{\epsilon}, T^{\epsilon}-T_{a p p}^{\epsilon}\right)\right\|_{L^{\infty}\left(0, S ; L^{2}(G)\right)} \leq C \epsilon^{\frac{3}{2}}
$$

for some positive constant $C$ independent of $\epsilon$.

REMARK 2.1. Noting that, in the above theorem 2.1, we only formulate the zero order compatibility conditions while any $m^{\text {th }}, m=0,1,2, \cdots$, order compatibility conditions can be obtained by the standard method, see, [7, 13, 16]. For example, taking $\partial_{t}$ of the boundary condition (1.11), using the equation (1.9) and the boundary condition (1.10), and then setting $z=0,1$, we get $\left.\Delta T_{0}^{0}\right|_{z=0,1}=0$. In this way, we can obtain the $1^{\text {th }}$ order compatibility conditions.

Noting that, due to the assumption $\left\|\left(u_{0 R}^{\epsilon}, T_{0 R}^{\epsilon}\right)(x, y, z)\right\|_{L^{2}(G)} \leq C \epsilon^{2}$, the convergence rate $O\left(\epsilon^{\frac{3}{2}}\right)$ is not optimal even though (2.19) has shown the convergence of the expansion up to the 1st order approximation. To get the optimal convergence rate, further assume that

$$
T_{0}^{0}(x, y, z)=1 \quad \text { near } \quad z=0, \quad \text { and } \quad T_{0}^{0}(x, y, z)=0 \quad \text { near } \quad z=1 .
$$

Then we have the optimal convergence rate $O\left(\epsilon^{2}\right)$. 
THEOREM 2.2. Let the assumptions of Theorem 2.1 hold. Furthermore, assume that (2.20) hold. Then, as $\epsilon \rightarrow 0$, for any $0<S<\infty$, we have the following convergence:

$$
\left\|\left(u^{\epsilon}-u_{a p p}^{\epsilon}, T^{\epsilon}-T_{a p p}^{\epsilon}-\epsilon^{2} \bar{T}^{2}\right)\right\|_{L^{\infty}\left(0, S ; L^{2}(G)\right)} \leq C \epsilon^{2}
$$

for some positive constant $C$ independent of $\epsilon$, where $\bar{T}^{2}=\bar{T}^{2}(x, y, z, \tau), \tau=\frac{t}{\epsilon}$, is the solution of the following linear problem

$$
\begin{aligned}
& \partial_{\tau} \bar{T}^{2}+\left(u_{0}^{0} \nabla\right) \bar{T}^{1}+\left(\bar{u}^{0} \nabla\right) T_{0}^{1}+\left(\bar{u}^{0} \nabla\right) \bar{T}^{1}+\left(\bar{u}^{1} \nabla\right) T_{0}^{0}+\left(\bar{u}^{0} \nabla\right) \partial_{t} T^{0}(t=0) \tau \\
& \quad=\Delta \bar{T}^{1},(x, y, z) \in G, \tau>0, \\
& \bar{T}^{2}(x, y, z, \tau \rightarrow \infty)=0,(x, y, z) \in G .
\end{aligned}
$$

REMARK 2.2. The convergence result (2.21) implies that

$$
\left\|\left(u^{\epsilon}-u_{\text {app }}^{\epsilon}, T^{\epsilon}-T_{a p p}^{\epsilon}\right)\right\|_{L^{\infty}\left(0, S ; L^{2}(G)\right)} \leq C \epsilon^{2}
$$

because $\left|\bar{T}^{2}\right| \leq C$ for some positive constant $C$, see $\S 3$.

REMARK 2.3. The assumption (2.20) is only a technical one, which guarantees that $\left.\bar{T}^{2}\left(x, y, z, \frac{t}{\epsilon}\right)\right|_{z=0,1}=0$ holds. Otherwise, the boundary layer occurs, and an extra correction term of boundary layer with two fast variables is needed to impose in the construction of approximating solution. Of course, this is interesting but very complicated and will be discussed in the future.

REMARK 2.4. The similar higher-order correction result in powers of $\epsilon$ can be obtained in the same way provided the initial data have such a higher order correction in powers of $\epsilon$ under the assumption of $T_{0}^{\epsilon}$ similar to (2.20).

\section{Approximating solution and method of asymptotic analysis}

In this section we construct the approximating solution including the initial layer expansion near $t=0$ and the outer one away from $t=0$. Some useful properties of this approximating solution are given. We start with the regular outer expansion.

3.1. Outer expansions. Away from the initial time $t=0$, the solution to the system (1.1)-(1.5) is expected to be well-approximated by the following expansion

$$
\left(u_{o u}^{\epsilon}, p_{o u}^{\epsilon}, T_{o u}^{\epsilon}\right)=\sum_{i=0}^{1} \epsilon^{i}\left(u^{i}, p^{i}, T^{i}\right)(x, y, z, t)
$$

with $\left(u^{i}, p^{i}, T^{i}\right)(x, y, z, t)$ to be determined later.

Inserting (3.1) into the system (1.1)-(1.5), and then comparing the coefficient of leading order $\epsilon^{0}$ and the first order $\epsilon^{1}$ in the resulting system, one can obtain:

(i) The leading order outer functions $\left(u^{0}, p^{0}, T^{0}\right)(x, y, z, t)$ satisfies so-called infinite Prandtl number system (1.7)-(1.11), namely,

$$
\begin{aligned}
& \nabla p^{0}+\frac{1}{E k} e_{3} \times u^{0}=\Delta u^{0}+\operatorname{Rae}_{3} T^{0}, \\
& \nabla \cdot u^{0}=0, \\
& \partial_{t} T^{0}+\left(u^{0} \cdot \nabla\right) T^{0}=\Delta T^{0}, \\
& \left.u^{0}\right|_{z=0,1}=0, \\
& \left.T^{0}\right|_{z=0}=1,\left.T^{0}\right|_{z=1}=0 .
\end{aligned}
$$


We impose the initial data as follws:

$$
T^{0}(t=0)=T_{0}^{0}(x, y, z)
$$

(ii) The first order outer function $\left(u^{1}, p^{1}, T^{1}\right)(x, y, z, t)$ satisfies the linearized infinite Prandtl number type system (2.3)-(2.7), namely,

$$
\begin{aligned}
& \nabla p^{1}+\frac{1}{E k} e_{3} \times u^{1}=\Delta u^{1}+\operatorname{Rae}_{3} T^{1}-\partial_{t} u^{0}-\left(u^{0} \cdot \nabla\right) u^{0} \\
& \nabla \cdot u^{1}=0 \\
& \partial_{t} T^{1}+\left(u^{0} \cdot \nabla\right) T^{1}+\left(u^{1} \cdot \nabla\right) T^{0}=\Delta T^{1} \\
& \left.u^{1}\right|_{z=0,1}=0 \\
& \left.T^{1}\right|_{z=0,1}=0
\end{aligned}
$$

We also impose the initial data as follows:

$$
T^{1}(t=0)=\tilde{T}_{0}^{1}(x, y, z)
$$

where $\tilde{T}_{0}^{1}(x, y, z)$ will be determined later (see below $(3.23)$ ) .

Noting that the infinite Prandtl number system (1.7)-(1.12) is one system of stationary Stokes equations with rotation and regarding time $t$ as a parameter coupled with heat advection-diffusion equations via a buoyancy force while the linearized infinite Prandtl number type system (2.3)-(2.8) is one linear system of Stokes equations coupled with a linearized heat advection-diffusion equations. Therefore the existence of the smooth solutions is the same as for the incompressible Stokes equations. We have:

Proposition 3.1. Assume that $T_{0}^{0}, \tilde{T}_{0}^{1} \in C^{\infty}(G)$ satisfy the suitable compatibility conditions like $\left.T_{0}^{0}\right|_{z=0}=1,\left.T_{0}^{0}\right|_{z=1}=0,\left.\tilde{T}_{0}^{1}\right|_{z=0,1}=0$, etc. Then there there exists a unique and global $C^{\infty}(G \times[0, \infty))$ smooth solution to the system (1.7)-(1.12) and (2.3)-(2.8) respectively.

Proof. The proof of Proposition 3.1 is elementary and we omit it.

After the determinations of these outer functions away from $t=0$, direct calculation shows that the outer solution $\left(u_{o u}^{\epsilon}, p_{o u}^{\epsilon}, T_{o u}^{\epsilon}\right)$ satisfies

$$
\begin{aligned}
& \epsilon\left[\partial_{t} u_{o u}^{\epsilon}+\left(u_{o u}^{\epsilon} \cdot \nabla\right) u_{o u}^{\epsilon}\right]+\nabla p_{o u}^{\epsilon}+\frac{1}{E k} e_{3} \times u_{o u}^{\epsilon}=\Delta u_{o u}^{\epsilon}+R a e_{3} T_{o u}^{\epsilon}+R_{o u, u}^{\epsilon}, \\
& \nabla \cdot u_{o u}^{\epsilon}=0 \\
& \partial_{t} T_{o u}^{\epsilon}+\left(u_{o u}^{\epsilon} \cdot \nabla\right) T_{o u}^{\epsilon}=\Delta T_{o u}^{\epsilon}+R_{o u, T}^{\epsilon} \\
& \left.u_{o u}^{\epsilon}\right|_{z=0,1}=0 \\
& \left.T_{o u}^{\epsilon}\right|_{z=0}=1,\left.T_{o u}^{\epsilon}\right|_{z=1}=0
\end{aligned}
$$

where the remainders $R_{o u, u}^{\epsilon}$ and $R_{o u, T}^{\epsilon}$ satisfy the estimates

$$
\left\|\left(R_{o u, u}^{\epsilon}, R_{o u, T}^{\epsilon}\right)\right\|_{L^{\infty}\left(0, S ; H^{s}(G)\right)} \leq C \epsilon^{2}
$$

only if the outer functions $\left(u^{i}, p^{i}, T^{i}\right)(x, y, z, t), i=0,1$, are given.

Now we turn to the construction of the initial layer functions. 
3.2. Initial layer expansion. Near $t=0$, we will approximate the solution uniformly up to $t=0$ by the following two-scale expansion

$$
\left(u_{a p p}^{\epsilon}, p_{a p p}^{\epsilon}, T_{a p p}^{\epsilon}\right)=\left(u_{o u}^{\epsilon}, p_{o u}^{\epsilon}, T_{o u}^{\epsilon}\right)(x, y, z, t)+\left(u_{I}^{\epsilon}, p_{I}^{\epsilon}, T_{I}^{\epsilon}\right)(x, y, z, \tau), \tau=\frac{t}{\epsilon},
$$

where $\left(u_{o u}^{\epsilon}, p_{o u}^{\epsilon}, T_{o u}^{\epsilon}\right)$ is given by (3.2)-(3.6) and

$$
\left(u_{I}^{\epsilon}, p_{I}^{\epsilon}, T_{I}^{\epsilon}\right)=\sum_{i=0}^{1} \epsilon^{i}\left(\bar{u}^{i}, \bar{p}^{i}, \bar{T}^{i}\right), \quad\left(\bar{u}^{i}, \bar{p}^{i}, \bar{T}^{i}\right)(\tau \rightarrow \infty)=0 .
$$

We can derive by the direct calculations that

$$
\begin{gathered}
\epsilon\left[\partial_{t} u_{a p p}^{\epsilon}+\left(u_{a p p}^{\epsilon} \cdot \nabla\right) u_{a p p}^{\epsilon}\right]+\nabla p_{a p p}^{\epsilon}+\frac{1}{E k} e_{3} \times u_{a p p}^{\epsilon}-\Delta u_{a p p}^{\epsilon}-R a e_{3} T_{a p p}^{\epsilon} \\
=R_{o u, u}^{\epsilon}+\left(\partial_{\tau} \bar{u}^{0}+\frac{1}{E k} e_{3} \times \bar{u}^{0}+\nabla \bar{p}^{0}-\Delta \bar{u}^{0}-R a e_{3} \bar{T}^{0}\right) \\
+\epsilon\left(\partial_{\tau} \bar{u}^{1}+\frac{1}{E k} e_{3} \times \bar{u}^{1}+\nabla \bar{p}^{1}-\Delta \bar{u}^{1}-R a e_{3} \bar{T}^{1}\right. \\
\left.+\left(\bar{u}^{0} \cdot \nabla\right) u_{0}^{0}+\left(u_{0}^{0} \cdot \nabla\right) \bar{u}^{0}+\left(\bar{u}^{0} \cdot \nabla\right) \bar{u}^{0}\right)+R_{I, u}^{\epsilon}, \\
\nabla \cdot u_{a p p}^{\epsilon}=\nabla \cdot u_{I}^{\epsilon}=\sum_{i=0}^{1} \epsilon^{i} \nabla \cdot \bar{u}^{i}, \\
\partial_{t} T_{a p p}^{\epsilon}+\left(u_{a p p}^{\epsilon} \cdot \nabla\right) T_{a p p}^{\epsilon}-\Delta T_{a p p}^{\epsilon} \\
=R_{o u, T}^{\epsilon}+\frac{1}{\epsilon} \partial_{\tau} \bar{T}^{0}+\left(\partial_{\tau} \bar{T}^{1}+\bar{u}^{0} \cdot \nabla T_{0}^{0}+\left(u^{0}+\bar{u}^{0}\right) \cdot \nabla \bar{T}^{0}\right)+R_{I, T}^{\epsilon}, \\
\left.u_{a p p}^{\epsilon}\right|_{z=0,1}=\left.u_{I}^{\epsilon}\right|_{z=0,1}=\left.\sum_{i=0}^{1} \epsilon^{i} \bar{u}^{i}\right|_{z=0,1}, \\
\left.T_{a p p}^{\epsilon}\right|_{z=0}=1+\left.T_{I}^{\epsilon}\right|_{z=0}=1+\left.\sum_{i=0}^{1} \epsilon^{i} \bar{T}^{i}\right|_{z=0}, \\
\left.T_{a p p}^{\epsilon}\right|_{z=1}=\left.T_{I}^{\epsilon}\right|_{z=1}=\left.\sum_{i=0}^{1} \epsilon^{i} \bar{T}^{i}\right|_{z=1}, \\
u_{a p p}^{\epsilon}(t=0)=\sum_{i=0}^{1} \epsilon^{i}\left(u^{i}(t=0)+\bar{u}^{i}(\tau=0)\right), \\
T_{a p p}^{\epsilon}(t=0)=\sum_{i=0}^{1} \epsilon^{i}\left(T^{i}(t=0)+\bar{T}^{i}(\tau=0)\right),
\end{gathered}
$$

where the remainders $R_{I, u}^{\epsilon}$ and $R_{I, T}^{\epsilon}$, caused by the initial layer, is given exactly by

$$
\begin{aligned}
R_{I, u}^{\epsilon}= & \epsilon^{2}\left(u_{0}^{0} \cdot \nabla \bar{u}^{1}+\bar{u}^{0} \cdot \nabla\left(\tau \partial_{t} u^{0}\left(x, s_{1}\right)+u^{1}(x, t)\right)+\bar{u}^{1} \cdot \nabla u_{0}^{0}\right. \\
& \left.+\bar{u}^{0} \cdot \nabla \bar{u}^{1}+\bar{u}^{1} \cdot \nabla \bar{u}^{0}+\left(\tau \partial_{t} u^{0}\left(x, s_{2}\right)+u^{1}(x, t)\right) \cdot \nabla \bar{u}^{0}\right) \\
& +\epsilon^{3}\left(\left(\tau \partial_{t} u^{0}\left(x, s_{3}\right)+u^{1}(x, t)\right) \cdot \nabla \bar{u}^{1}\right. \\
& \left.+\bar{u}^{1} \cdot \nabla\left(\tau \partial_{t} u^{0}\left(x, s_{4}\right)+u^{1}(x, t)\right)+\bar{u}^{1} \cdot \nabla \bar{u}^{1}\right), \\
& 0<s_{i}<t, i=1,2,3,4
\end{aligned}
$$


and

$$
R_{I, T}^{\epsilon}=R_{I, T 1}^{\epsilon}+R_{I, T 2}^{\epsilon}
$$

with

$$
\begin{aligned}
R_{I, T 1}^{\epsilon}= & \epsilon\left(\left(\left(u^{1}+\bar{u}^{1}\right) \cdot \nabla\right) \bar{T}^{0}+\left(u_{0}^{0} \nabla\right) \bar{T}^{1}+\left(\bar{u}^{0} \nabla\right) T_{0}^{1}+\left(\bar{u}^{0} \nabla\right) \bar{T}^{1}\right. \\
& \left.+\left(\bar{u}^{1} \nabla\right) T_{0}^{0}+\left(\bar{u}^{0} \nabla\right) \partial_{t} T^{0}(t=0) \tau-\Delta \bar{T}^{1}\right)
\end{aligned}
$$

and

$$
\begin{aligned}
R_{I, T 2}^{\epsilon}= & \epsilon^{2}\left(\left(\tau \partial_{t} u^{0}\left(x, s_{5}\right)+u_{0}^{1}\right) \cdot \nabla \bar{T}^{1}+\tau^{2} \bar{u}^{0} \cdot \nabla\left(\frac{1}{2} \partial_{t t} T^{0}\left(x, s_{6}\right)+\partial_{t} T^{1}\left(x, s_{7}\right)\right)\right. \\
+ & \left.+\bar{u}^{1} \cdot \nabla\left(\tau \partial_{t} T^{0}(t=0)+T^{1}(t=0)+\bar{T}^{1}\right)\right) \\
& 0<s_{i}<t, i=5,6,7
\end{aligned}
$$

Now we can obtain the systems being satisfied by the initial layer functions by setting the coefficients of order $O\left(\epsilon^{k}\right)$ in the system (3.10)-(3.12) as zero and requiring that the approximating solution satisfies the boundary and initial conditions.

First taking the coefficient of order $\epsilon^{-1}$ in (3.12) as zero, one has

$$
\partial_{\tau} \bar{T}^{0}=0,
$$

which, together with $\bar{T}^{0}(\tau \rightarrow \infty)=0$ in $(3.9)$, yields to $(2.9)$, i.e.,

$$
\bar{T}^{0}(x, y, z, \tau)=0 \text {. }
$$

This does also show that the temperature has no zero order initial layer.

Then, setting the coefficients of order $\epsilon^{0}$ in the system (3.10)-(3.12) as zero, using (2.9) and requiring that the approximating solution satisfies the boundary and initial conditions, it follows from (3.10)-(3.17) that the initial layer functions $\left(\bar{u}^{0}, \bar{p}^{0}, \bar{T}^{1}\right)$ satisfy the system $(2.10)-(2.14)$.

It follows from the equation $(2.12)$ of $\bar{T}^{1}$ and the decay condition $\bar{T}^{1}(\tau \rightarrow \infty)=0$ in (3.9) that

$$
\bar{T}^{1}=-\int_{\tau}^{\infty}\left(\bar{u}^{0} \cdot \nabla T_{0}^{0}\right)(s) d s .
$$

Using the boundary condition (2.13) and the equation (3.22), one gets

$$
\left.\bar{T}^{1}\right|_{z=0,1}=0 .
$$

After the determination of $\bar{T}^{1}$, we require to take the initial data $\tilde{T}_{0}^{1}$ of $T^{1}$ as

$$
T^{1}(t=0)=\tilde{T}_{0}^{1}=T_{0}^{1}-\bar{T}^{1}(\tau=0) .
$$

Thus, the initial condition (2.8) is obtained from (3.23).

Finally, as in the above, it follows from the order $\epsilon^{1}$ in (3.10)-(3.11), (3.13) and (3.16) that $\left(\bar{u}^{1}, \bar{p}^{1}\right)$ satisfy the system (2.15)-(2.18).

Now we state the decay properties of the initial layer functions.

Proposition 3.2. Let the assumptions of Theorem 2.1 hold. Then there exist a unique and smooth solution $\left(\bar{u}^{0}, \bar{p}^{0}, \bar{T}^{1}\right)$ to the system (2.10)-(2.14) and a unique and 
smooth solution $\left(\bar{u}^{1}, \bar{p}^{1}\right)$ to the system (2.15)-(2.18) satisfying the exponential decay to zero as $\tau \rightarrow \infty$, namely,

$$
\left\|\left(\bar{u}^{0}, \bar{T}^{1}, \bar{u}^{1}\right)(\cdot, \tau)\right\|_{H^{s}(G)} \leq C e^{-\alpha \tau}
$$

for some positive constants $C, \alpha$ and any $s \geq 1$.

To prove Proposition 3.2, we recall the following lemma about the Stokes operator $[5,13]$.

LEMma 3.3. For the stationary Stokes equations

$$
\begin{aligned}
-\Delta U+\nabla q & =f(x, y, z),(x, y, z) \in G, \\
\nabla \cdot U & =0,(x, y, z) \in G, \\
\left.U\right|_{z=0,1} & =0, U \quad \text { is periodic with respect to } x \text { and } y,
\end{aligned}
$$

we have that, if $f \in H^{l}(G)$, then the following estimate holds:

$$
\|U\|_{H^{l+2}(G)}+\|\nabla q\|_{H^{l}(G)} \leq C\|f\|_{H^{l}(G)},
$$

where $C$ depends only upon the domain $G$.

Proof of Proposition 3.2. We only prove the exponential decay rate of $\bar{u}^{0}$ in (3.24) because the others can be easily obtained in similar way by the equation (3.22) and the system (2.15)-(2.18) and the exponential decay rate of $\bar{u}^{0}$.

Taking $\partial_{\tau}^{s}$ of (2.10), multiplying the resulting equation by $\partial_{\tau}^{s} \bar{u}^{0}$ and integrating over $G$ with respect to $(x, y, z)$, by integration by parts, one gets

$$
\frac{1}{2} \frac{d}{d \tau}\left\|\partial_{\tau}^{s} \bar{u}^{0}(\tau)\right\|_{L^{2}(G)}^{2}+\left\|\nabla \partial_{\tau}^{s} \bar{u}^{0}(\tau)\right\|_{L^{2}}^{2}=0
$$

due to $\left.\partial_{\tau}^{s} \bar{u}^{0}\right|_{z=0,1}=0$.

Using the Poincaré's inequality, one has

$$
\frac{d}{d \tau}\left\|\partial_{\tau}^{s} \bar{u}^{0}(\tau)\right\|_{L^{2}(G)}^{2}+C\left\|\partial_{\tau}^{s} \bar{u}^{0}(\tau)\right\|_{L^{2}}^{2} \leq 0
$$

for some positive constant $C$. This yields to that

$$
\left\|\partial_{\tau}^{s} \bar{u}^{0}(\tau)\right\|_{L^{2}(G)} \leq C e^{-C \tau}
$$

Noting that $w(\tau)=\partial_{\tau}^{s} \bar{u}^{0}$ satisfies

$$
\begin{aligned}
& \partial_{\tau} w+\frac{1}{E k} e_{3} \times w+\nabla \partial_{\tau}^{s} \bar{p}^{0}=\Delta w, \\
& \nabla \cdot w=0, \\
& \left.w\right|_{z=0,1}=0, \\
& w(\tau=0)=w_{0}
\end{aligned}
$$

Applying lemma 3.3 to the system (3.26)-(3.29), one has, for any $l>0$,

$$
\|w(\tau)\|_{H^{l+2}(G)} \leq C\left(\left\|\partial_{\tau} w(\tau)\right\|_{H^{l}(G)}+\frac{1}{E k}\|w(\tau)\|_{H^{l}(G)}\right) .
$$

Using the estimate (3.30) repeatedly and (3.25), one has, for any $s \geq 1$,

$$
\left\|\bar{u}^{0}\right\|_{H^{s}(G)} \leq C \sum_{i=0}^{s}\left\|\partial_{\tau}^{i} \bar{u}^{0}\right\|_{L^{2}(G)} \leq C e^{-C \tau} .
$$

The proof of Proposition 3.2 is complete. 
3.3. Approximating solutions. With outer functions and initial layer functions defined in section 3.1 and 3.2 , we can define the desired uniformly-valid approximating solution to the system (1.1)-(1.6). Then our previous computations show that $\left(u_{a p p}^{\epsilon}, p_{a p p}^{\epsilon}, T_{a p p}^{\epsilon}\right)$ solves the following initial-boundary problem:

$$
\begin{aligned}
& \epsilon\left[\partial_{t} u_{a p p}^{\epsilon}+\left(u_{a p p}^{\epsilon} \cdot \nabla\right) u_{a p p}^{\epsilon}\right]+\nabla p_{a p p}^{\epsilon}+\frac{1}{E k} e_{3} \times u_{a p p}^{\epsilon} \\
& \quad=\Delta u_{a p p}^{\epsilon}+R a e_{3} T_{a p p}^{\epsilon}+R_{o u, u}^{\epsilon}+R_{I, u}^{\epsilon}, \\
& \nabla \cdot u_{a p p}^{\epsilon}=0, \\
& \partial_{t} T_{a p p}^{\epsilon}+\left(u_{a p p}^{\epsilon} \cdot \nabla\right) T_{a p p}^{\epsilon}=\Delta T_{a p p}^{\epsilon}+R_{o u, T}^{\epsilon}+R_{I, T}^{\epsilon}, \\
& \left.u_{a p p}^{\epsilon}\right|_{z=0,1}=0, \\
& \left.T_{a p p}^{\epsilon}\right|_{z=0}=1,\left.T_{a p p}^{\epsilon}\right|_{z=1}=0, \\
& \left(u_{a p p}^{\epsilon}, T_{a p p}^{\epsilon}\right)(t=0)=\left(u_{0}^{0}+\epsilon u_{0}^{1}, T_{0}^{0}+\epsilon T_{0}^{1}\right),
\end{aligned}
$$

where the remainders $R_{o u, u}^{\epsilon}, R_{o u, T}^{\epsilon}$ satisfy the estimate (3.7) and $R_{I, u}^{\epsilon}, R_{I, T}^{\epsilon}$ defined by (3.18) and (3.19) respectively satisfy the the following estimate

$$
\left\|R_{I, u}^{\epsilon}(t)\right\|_{L^{\infty}(G)} \leq C \epsilon^{2}(\tau+1) e^{-\alpha \tau},\left\|R_{I, T}^{\epsilon}(t)\right\|_{L^{\infty}(G)} \leq C \epsilon\left(\tau^{2}+\tau+1\right) e^{-\alpha \tau}
$$

for some positive constants $C$ and $\alpha$ and for any $t \in[0, S]$ and any fixed $S>0$. The estimate (3.36) can easily obtained by the definitions of $R_{I, u}^{\epsilon}, R_{I, T}^{\epsilon}$ and the decay estimate (3.24).

We now turn to the proof of convergence rate.

\section{The Proof of Main Results}

In this section, we will prove Theorems 2.1 and 2.2 by the careful energy method. In the following denote $C$ by a positive generic constant independent of $\epsilon$. Noting that $C$ may depend upon $S$ for any fixed $S>0$. Let $t \in[0, S]$. We start with the proof of convergence rate $O\left(\epsilon^{\frac{3}{2}}\right)$.

4.1. Convergence rate $O\left(\epsilon^{\frac{3}{2}}\right)$. In this subsection we assume that (2.1) holds. We will prove Theorem 2.1.

Let $\left(u^{\epsilon}, p^{\epsilon}, T^{\epsilon}\right)$ be the global weak solution to (1.1)-(1.6) in the Leray's sense, see $[19,20]$. Let $\left(u_{a p p}^{\epsilon}, p_{a p p}^{\epsilon}, T_{a p p}^{\epsilon}\right)$ be the approximating solution constructed in section $\S 3$. Set $\left(u_{R}^{\epsilon}, p_{R}^{\epsilon}, T_{R}^{\epsilon}\right)=\left(u^{\epsilon}-u_{a p p}^{\epsilon}, p^{\epsilon}-p_{a p p}^{\epsilon}, T^{\epsilon}-T_{a p p}^{\epsilon}\right)$. Then it follows from (1.1)-(1.6) and (3.31)-(3.35) that $\left(u_{R}^{\epsilon}, p_{R}^{\epsilon}, T_{R}^{\epsilon}\right)$ satisfies the following 'error' equations

$$
\begin{aligned}
& \epsilon\left[\partial_{t} u_{R}^{\epsilon}+\left(u_{a p p}^{\epsilon} \cdot \nabla\right) u_{R}^{\epsilon}+\left(u_{R}^{\epsilon} \cdot \nabla\right)\left(u_{a p p}^{\epsilon}+u_{R}^{\epsilon}\right)\right]+\nabla p_{R}^{\epsilon}+\frac{1}{E k} e_{3} \times u_{R}^{\epsilon} \\
& =\Delta u_{R}^{\epsilon}+\operatorname{Rae}_{3} T_{R}^{\epsilon}-R_{o u, u}^{\epsilon}-R_{I, u}^{\epsilon}, \\
& \nabla \cdot u_{R}^{\epsilon}=0 \\
& \partial_{t} T_{R}^{\epsilon}+\left(u_{a p p}^{\epsilon} \cdot \nabla\right) T_{R}^{\epsilon}+\left(u_{R}^{\epsilon} \cdot \nabla\right)\left(T_{a p p}^{\epsilon}+T_{R}^{\epsilon}\right)=\Delta T_{R}^{\epsilon}-R_{o u, T}^{\epsilon}-R_{I, T}^{\epsilon}, \\
& \left.u_{R}^{\epsilon}\right|_{z=0,1}=0 \text {, } \\
& \left.T_{R}^{\epsilon}\right|_{z=0,1}=0 \text {, } \\
& u_{R}^{\epsilon}(t=0)=u_{R 0}^{\epsilon}(x, y, z), T_{R}^{\epsilon}(t=0)=T_{0 R}^{\epsilon}(x, y, z)
\end{aligned}
$$

Multiplying (4.3) by $T_{R}^{\epsilon}$ and integrating over $G$ with respect to $(x, y, z)$, by integration by parts, Cauchy-Schwartz's inequality, using the properties of the approximating 
solution, the equation (4.2), the boundary condition (4.5) and the estimates (3.7) and (3.36), one gets

$$
\begin{aligned}
& \frac{1}{2} \frac{d}{d t}\left\|T_{R}^{\epsilon}\right\|_{L^{2}(G)}^{2}+\left\|\nabla T_{R}^{\epsilon}\right\|_{L^{2}(G)}^{2} \\
= & -\int_{G}\left(u_{R}^{\epsilon} \cdot \nabla\right) T_{a p p}^{\epsilon} T_{R}^{\epsilon} d x d y d z-\int_{G}\left(R_{o u, T}^{\epsilon}+R_{I, T}^{\epsilon}\right) T_{R}^{\epsilon} d x d y d z \\
\leq & \delta\left\|T_{R}^{\epsilon}\right\|_{L^{2}(G)}^{2}+C\left\|u_{R}^{\epsilon}\right\|_{L^{2}(G)}^{2}+C \epsilon^{4}+C \epsilon^{2}\left(\tau^{2}+\tau+1\right)^{2} e^{-2 \alpha \tau} .
\end{aligned}
$$

Here we have used the estimate

$$
\left\|R_{o u, T}^{\epsilon}+R_{I, T}^{\epsilon}\right\|_{L^{2}(G)} \leq C \epsilon^{2}+C \epsilon\left(\tau^{2}+\tau+1\right) e^{-\alpha \tau}
$$

on the remainder $R_{\text {ou,T }}^{\epsilon}+R_{I, T}^{\epsilon}$.

Applying the Poincaré's inequality and taking $\delta$ to be sufficiently small but independent of $\epsilon$, one gets

$$
\begin{aligned}
& \frac{d}{d t}\left\|T_{R}^{\epsilon}\right\|_{L^{2}(G)}^{2}+\left\|\nabla T_{R}^{\epsilon}\right\|_{L^{2}(G)}^{2} \\
\leq & C\left\|u_{R}^{\epsilon}\right\|_{L^{2}(G)}^{2}+C \epsilon^{4}+C \epsilon^{2}\left(\tau^{2}+\tau+1\right)^{2} e^{-2 \alpha \tau} .
\end{aligned}
$$

Integrating (4.8) with respect to $t$ over $[0, t]$ for any $t \in[0, S]$ and any fixed $S>0$, one gets

$$
\begin{gathered}
\left\|T_{R}^{\epsilon}(t)\right\|_{L^{2}(G)}^{2}+\int_{0}^{t}\left\|\nabla T_{R}^{\epsilon}(t)\right\|_{L^{2}(G)}^{2} d t \\
\leq\left\|T_{R}^{\epsilon}(t=0)\right\|_{L^{2}(G)}^{2}+C \int_{0}^{t}\left\|u_{R}^{\epsilon}(t)\right\|_{L^{2}(G)}^{2} d t+C \epsilon^{3}
\end{gathered}
$$

due to the fact that

$$
\int_{0}^{t}\left(\tau^{2}+\tau+1\right)^{2} e^{-2 \alpha \tau} d t \leq C \epsilon .
$$

Multiplying (4.1) by $u_{R}^{\epsilon}$ and integrating over $G$ with respect to $(x, y, z)$, by integration by parts, Cauchy-Schwartz's inequality, using the properties of the approximating solution, the equation (4.2), the boundary condition (4.4) and the estimates (3.7) and (3.36), one gets

$$
\begin{aligned}
& \frac{\epsilon}{2} \frac{d}{d t}\left\|u_{R}^{\epsilon}\right\|_{L^{2}(G)}^{2}+\left\|\nabla u_{R}^{\epsilon}\right\|_{L^{2}(G)}^{2} \\
= & -\epsilon \int_{G}\left(u_{R}^{\epsilon} \cdot \nabla\right) u_{a p p}^{\epsilon} u_{R}^{\epsilon} d x d y d z+R a \int_{G} e_{3} T_{R}^{\epsilon} u_{R}^{\epsilon} d x d y d z-\int_{G}\left(R_{o u, u}^{\epsilon}+R_{I, u}^{\epsilon}\right) u_{R}^{\epsilon} d x d y d z \\
\leq & \epsilon\left\|\nabla u_{a p p}^{\epsilon}\right\|_{L^{\infty}(G)}\left\|u_{R}^{\epsilon}(t)\right\|_{L^{2}(G)}^{2}+\delta\left\|u_{R}^{\epsilon}\right\|_{L^{2}(G)}^{2}+C R a^{2}\left\|T_{R}^{\epsilon}\right\|_{L^{2}(G)}^{2} \\
& +C \epsilon^{4}+C \epsilon^{4}(\tau+1)^{2} e^{-2 \alpha \tau} .
\end{aligned}
$$

Applying the Poincaré's inequality, restricting $\epsilon$ to be sufficiently small such that $\epsilon\left\|\nabla u_{a p p}^{\epsilon}\right\|_{L^{\infty}(G)} \leq C \epsilon \leq \frac{1}{4}$ and taking $\delta$ to be sufficiently small $\left(\delta=\frac{1}{4}\right)$ but independent of $\epsilon$, one gets

$$
\epsilon \frac{d}{d t}\left\|u_{R}^{\epsilon}(t)\right\|_{L^{2}(G)}^{2}+\left\|u_{R}^{\epsilon}(t)\right\|_{L^{2}(G)}^{2} \leq C R a^{2}\left\|T_{R}^{\epsilon}(t)\right\|_{L^{2}(G)}^{2}+C \epsilon^{4},
$$


i.e.

$$
\frac{d}{d t}\left(e^{\frac{t}{\epsilon}}\left\|u_{R}^{\epsilon}(t)\right\|_{L^{2}(G)}^{2}\right) \leq\left(C R a^{2}\left\|T_{R}^{\epsilon}(t)\right\|_{L^{2}(G)}^{2}+C \epsilon^{4}\right) \frac{1}{\epsilon} e^{\frac{t}{\epsilon}} .
$$

Integrating (4.10) with respect to $t$ over $[0, t]$ for any $t \in[0, S]$ and any fixed $S>0$, one gets

$$
\left\|u_{R}^{\epsilon}(t)\right\|_{L^{2}(G)}^{2} \leq\left\|u_{R}^{\epsilon}(t=0)\right\|_{L^{2}(G)}^{2}+C R a^{2}\left\|T_{R}^{\epsilon}(t)\right\|_{L^{\infty}\left((0, t) ; L^{2}(G)\right)}^{2}+C \epsilon^{4} .
$$

Combining (4.9) and (4.11), one gets

$$
\left\|u_{R}^{\epsilon}(t)\right\|_{L^{2}(G)}^{2} \leq C\left\|\left(u_{R}^{\epsilon}, T_{R}^{\epsilon}\right)(t=0)\right\|_{L^{2}(G)}^{2}+C\left\|u_{R}^{\epsilon}(t)\right\|_{L^{2}\left((0, t) ; L^{2}(G)\right)}^{2}+C \epsilon^{3} .
$$

Using Gronwall's lemma and the assumptions on the 'error' of initial data, i.e., $\left\|\left(u_{R}^{\epsilon}, T_{R}^{\epsilon}\right)(t=0)\right\|_{L^{2}(G)}^{2}=\left\|\left(u_{0 R}^{\epsilon}, T_{0 R}^{\epsilon}\right)\right\|_{L^{2}(G)}^{2} \leq C \epsilon^{4}$, one gets

$$
\left\|u_{R}^{\epsilon}(t)\right\|_{L^{2}\left((0, t) ; L^{2}(G)\right)}^{2} \leq C \epsilon^{3},
$$

and, hence, one has

$$
\left\|u_{R}^{\epsilon}(t)\right\|_{L^{\infty}\left((0, t) ; L^{2}(G)\right)}^{2} \leq C \epsilon^{3} .
$$

Inserting (4.12) into (4.9) and using the assumptions on the 'error' of initial data again, one gets

$$
\left\|T_{R}^{\epsilon}(t)\right\|_{L^{2}(G)}^{2} \leq C \epsilon^{3} .
$$

Therefore, it follows from (4.14) that

$$
\left\|T_{R}^{\epsilon}(t)\right\|_{L^{\infty}\left((0, t) ; L^{2}(G)\right.}^{2} \leq C \epsilon^{3} .
$$

The estimates (4.13) and (4.15) yields to the desired estimate (2.19) in Theorem 2.1.

The proof of Theorem 2.1 is complete.

Obviously, the convergence rate $O\left(\epsilon^{\frac{3}{2}}\right)$ is not optimal according to the assumption on initial data. In the following subsection we obtain the optimal convergence rate $O\left(\epsilon^{2}\right)$ under further assumption on initial data.

4.2. Optimal convergence rate $O\left(\epsilon^{2}\right)$. In this subsection we assume that (2.1) and (2.20) hold. We will prove Theorem 2.2.

To get the optimal convergence rate $O\left(\epsilon^{2}\right)$, we need to cancel the order $O(\epsilon)$ term $R_{I, T 1}^{\epsilon}$, given by (3.20), in the remainder $R_{I, T}^{\epsilon}$ by introducing another initial layer function $\bar{T}^{2}$. We define $\bar{T}^{2}$ to be the solution of the system (2.22)-(2.23), which can be solved by

$$
\begin{aligned}
\bar{T}^{2}(\tau)= & -\int_{\tau}^{\infty}\left[\Delta \bar{T}^{1}-\left(\left(u_{0}^{0} \nabla\right) \bar{T}^{1}+\left(\bar{u}^{0} \nabla\right) T_{0}^{1}+\left(\bar{u}^{0} \nabla\right) \bar{T}^{1}\right.\right. \\
& \left.\left.+\left(\bar{u}^{1} \nabla\right) T_{0}^{0}+\left(\bar{u}^{0} \nabla\right) \partial_{t} T^{0}(t=0) \tau\right)\right](\tau) d \tau .
\end{aligned}
$$

Thus, we have

$$
\left.\bar{T}^{2}\right|_{z=0,1}=0 .
$$


In fact, the assumption (2.20) and the definition (3.22) of $\bar{T}^{1}$ give

$$
\bar{T}^{1}=0, \quad \text { near } \quad z=0,1,
$$

which, together with (4.16) and the boundary condition $\left.\left(\bar{u}^{0}, \bar{u}^{1}\right)\right|_{z=0,1}=0$, yields to the boundary condition (4.17).

By the exponential decay of the initial layer functions $\bar{u}^{0}, \bar{u}^{1}, \bar{T}^{1}$, one have

$$
\left\|\bar{T}^{2}(\tau)\right\|_{H^{s}(G)} \leq C e^{-\beta \tau} .
$$

Now set $\tilde{T}_{R}^{\epsilon}=T_{R}^{\epsilon}-\epsilon^{2} \bar{T}^{2}=T^{\epsilon}-T_{a p p}^{\epsilon}-\epsilon^{2} \bar{T}^{2}$. Then $\left(u_{R}^{\epsilon}, p_{R}^{\epsilon}, \tilde{T}_{R}^{\epsilon}\right)$ satisfies the following modified 'error' equations

$$
\begin{aligned}
& \epsilon\left[\partial_{t} u_{R}^{\epsilon}+\left(u_{a p p}^{\epsilon} \cdot \nabla\right) u_{R}^{\epsilon}+\left(u_{R}^{\epsilon} \cdot \nabla\right)\left(u_{a p p}^{\epsilon}+u_{R}^{\epsilon}\right)\right]+\nabla p_{R}^{\epsilon}+\frac{1}{E k} e_{3} \times u_{R}^{\epsilon} \\
& \quad=\Delta u_{R}^{\epsilon}+R a e_{3} \tilde{T}_{R}^{\epsilon}-R_{o u, u}^{\epsilon}-R_{I, u}^{\epsilon}+R a e_{3} \epsilon^{2} \bar{T}^{2}, \\
& \nabla \cdot u_{R}^{\epsilon}=0 \\
& \partial_{t} \tilde{T}_{R}^{\epsilon}+\left(u_{a p p}^{\epsilon} \cdot \nabla\right) \tilde{T}_{R}^{\epsilon}+\left(u_{R}^{\epsilon} \cdot \nabla\right)\left(T_{a p p}^{\epsilon}+\epsilon^{2} \bar{T}^{2}+\tilde{T}_{R}^{\epsilon}\right) \\
& \quad=\Delta \tilde{T}_{R}^{\epsilon}-R_{o u, T}^{\epsilon}-R_{I, T 2}^{\epsilon}-\epsilon^{2} u_{a p p}^{\epsilon} \cdot \nabla \bar{T}^{2}+\epsilon^{2} \Delta \bar{T}^{2}, \\
& \left.u_{R}^{\epsilon}\right|_{z=0,1}=0 \\
& \left.\tilde{T}_{R}^{\epsilon}\right|_{z=0,1}=0 \\
& u_{R}^{\epsilon}(t=0)=u_{R 0}^{\epsilon}(x, y, z), \tilde{T}_{R}^{\epsilon}(t=0)=T_{0 R}^{\epsilon}(x, y, z)-\epsilon^{2} \bar{T}^{2}(\tau=0) .
\end{aligned}
$$

Using the decay property (4.18) of $\bar{T}^{2}$ and the definition (3.21) of $R_{I, T 2}^{\epsilon}$, one has the following estimates on the remainder $-R_{o u, T}^{\epsilon}-R_{I, T 2}^{\epsilon}-\epsilon^{2} u_{a p p}^{\epsilon} \cdot \nabla \bar{T}^{2}+\epsilon^{2} \Delta \bar{T}^{2}$ appearing the system (4.21):

$$
\left\|-R_{o u, T}^{\epsilon}-R_{I, T 2}^{\epsilon}-\epsilon^{2} u_{a p p}^{\epsilon} \cdot \nabla \bar{T}^{2}+\epsilon^{2} \Delta \bar{T}^{2}\right\|_{L^{2}(G)} \leq C \epsilon^{2} .
$$

Noting that the estimate (4.25) is much better than the estimate (4.7). Now replacing the estimate (4.7) by the estimate (4.25) in subsection $\S 4.1$, then, as in the proof of Theorem 2.1, we can easily obtain the desired optimal convergence rate $O\left(\epsilon^{2}\right)$ in Theorem 2.2.

The proof of Theorem 2.2 is complete.

Acknowledgement. The authors would like to give many thanks to the referees for their constructive suggestions and comments. The first author would like to express his gratitude for the hospitality of College of Applied Sciences in Beijing University of Technology, when he visited there from September 2005 to June 2006.

\section{REFERENCES}

[1] E. Bodenschatz, W. Pesch and G. Ahlers, Recent developments in Rayleigh-Bénard convection, Annual Review of Fluid Mechanics, Annual Reviews, Palo Alto, Calif., 32, 709-778, 2000.

[2] P. Constantin and C. R. Doering, Infinite Prandtl number convection, J. Stat. Phys., 94, 159$172,1999$.

[3] P. Constantin and C. Foias, Navier-Stokes Equations, University of Chicago Press, 1988.

[4] P. Constantin, C. Hallstrom and V. Poutkaradze, Logarithmic bounds for infinite Prandtl number rotating convection, J. Math. Phys., 42, 773-783, 2001.

[5] P. Constantin, C. Hallstrom and V. Poutkaradze, Heat transport rotating convection, Physica D, 125, 275-284, 1999. 
[6] C. R. Doering and P. Constantin, On upper bounds for infinite Prandtl number convection with or without rotation, J. Math. Phys., 42, 784-795, 2001.

[7] L. C. Evans, Partial Differential Equations, Graduate Studies in Mathematics, American Mathematical Society, Providence, R. I., 19, 1998.

[8] C. Foias, O. Manley and R. Temam, Attractors for the Bénard problem: existence and physical bounds on their fractal dimension, Nonlinear Anal., TMA, 11, 939-967, 1987.

[9] S. Klainerman and A. Majda, Singular limits of quasilinear hyperbolic systems with large parameters and the incompressible limit of compressible fluids, Comm. Pure Appl. Math., 34, 481-524, 1981.

[10] J. L. Lions, Perturbations Singuliéres Dans les Problémes aux Limites et en Controle Optimal, Lecture Notes in Math., Springer-Verlag, New York, 323, 1973.

[11] H. V. Ly and E. S. Titi, Global Gevrey regularity for the Bénard convection in a porous medium with zero Darcy-Prandtl number, J. Nonlinear Sci., 9, 333-362, 1999.

[12] A. Majda, Introduction to PDEs and Waves for the Atmosphere and Ocean, Courant Lecture Notes in Mathematics, 9, New York University, Courant Institute of Mathematical Sciences, New York; American Mathematical Society, Providence, R. I., 2003.

[13] O. A. Ladyzhenskaya, The Mathematical Theory of Viscous Incompressible Flows, Gordon and Breach, London, New York, 1969.

[14] J Park, Dynamic bifurcation theory of Rayleigh-Bénard convection with infite Prandtl number, Discrete Contin. Dyn. Syst. Ser. B, 6, 591-604, 2006.

[15] E. D. Siggia, High Rayleigh number convection, Annual Review of Fluid Mechanics, Annual Reviews, Palo Alto, Calif., 26, 137-168, 1994.

[16] R. Temam, Navier-Stokes Equations, Theory and Numerical Analysis, reprint of the 1984 edition, AMS Chelsea, Providence, R. I., 2001.

[17] D. J. Tritton, Physical Fluid Dynamics, 2nd ed. Oxford Science Publication, Carendon, Oxford University Press, New York, 1988.

[18] M. I. Vishik and L. A. Lyusternik, Regular degeneration and boundary layer for linear differential equations with small paramater, Uspekki Mat. Nauk, 12, 3-122, 1957.

[19] X. M. Wang, Infinite Prandtl number limit of Rayleigh-Bénard convection, Comm. Pure Appl. Math., LVII, 1265-1282, 2004

[20] X. M. Wang, Large Prandtl number limit of the Boussinesq system of Rayleigh-Bénard convection, Appl. Math. Letters, 17, 821-825, 2004.

[21] Z. P. Xin, Viscous boundary layers and their stability (I), J. Part. Diff. Eqs., 11, 74-124, 1998. 